\title{
USE OF A PHASE TRANSITION CONCEPT FOR TRAFFIC FLOW CONDITION ESTIMATION
}

\author{
Oleg N. Larin ${ }^{1}$, Victor A. Dosenko ${ }^{2}$ \\ ${ }^{1}$ Moscow State University of Railway transport (MIIT), \\ 127994, Moscow, Avenue Obraztsov, 9 , \\ Phone: (916)617-67-60,e-mail: larin_on@mail.ru, \\ ${ }^{2}$ International Transport Academy, \\ Russia, 119019 Moscow, Gogolevskyi Boulevard 33/1 off. 15, \\ Phone: +79859216847, e-mail:dosenko@itamain.com
}

\begin{abstract}
The article covers the main models of traffic flow conditions, analyzes the condition estimation criteria, and provides the classification of models. The article provides the grounds for the use of the phase transition concept for traffic flow condition estimation. The models of the aggregate condition of free and congested traffic have been developed, the phase boundaries between free and congested traffic have been defined. Applicability conditions for the models of the aggregate condition of have been analyzed.
\end{abstract}

Keywords: traffic flow, classification condition, phase transitions

\section{Introduction}

The development transit networks projects should include the influence of network configuration on the efficiency of transit traffic [1]. Road network reconfiguration makes an impact on the characteristics of the traffic flow. The dependencies of transportation efficiency on the traffic flow parameters have been analyzed in numerous studies. To reflect these dependencies, the concept of traffic flow condition is used in the studies. Traffic flow condition is a highly aggregated qualitative characteristic of a traffic flow. Every traffic flow condition is associated with a certain level of economic efficiency of transportation, convenience from a driver's point of view, traffic safety, etc. The existence of traffic flow qualities is proven by experimental data $[2,3,4,5,11]$.

The qualities are sorted out on the basis of quantitative evaluation of a traffic flow according to certain criteria. The issues of traffic flow condition estimation have been analyzed in many studies by Russian and foreign authors. The present article covers the applicability of a phase transition concept for traffic flow condition estimation.

\section{Traffic flow condition models and their classification}

Different models, which can be grouped according to different properties, are used for the estimation of traffic flow condition. For example, depending on the number of traffic flow parameters that are used as traffic flow condition estimation criteria, single-criterion and multi-criteria models can be defined.

The advantage of the single-criterion traffic flow condition models consists in the simplicity of the estimation. However, these models cannot always adequately represent the real situation on the road. For this reason, multi-criteria models that suppose the comprehensive evaluation of a traffic flow condition on the basis of a combination of traffic flow parameters, are seen as more efficient for practical tasks. These models enable to take into consideration the reciprocal influence of multiple parameters on the flow condition. In fact, multi-criteria models broaden the range of traffic flow condition. The number of these conditions can increase depending on the number of the criteria that have been used for the evaluation. In multi-criteria models the influence of a parameter evolution is geared to the evolution of other parameters.

One of the most widely used models is the single-criterion model, «congested - non-congested traffic». In other studies, the following similar terms are used for the same model: «forced traffic - free traffic», «steady mode - unsteady mode», etc. The main criteria that enables to define such condition is 
traffic flow density. Once traffic flow reaches a certain density, the car speed is no longer defined by a driver's strategy - instead, it starts to be determined by the shared general conditions that are typical for congested traffic.

In theory, the average car speed in «non-congested traffic» is limited by the restrictions caused by road factors. Determinate (descending) functional dependency of average speed, $v$, on the flow density, $\rho$, is typical for congested traffic. In modern conditions and in the context of intense automobilization, numerous studies cover the dependencies of traffic flow parameters establishment in congested traffic.

In some single-criterion models traffic flow condition is treated as a function of road workload. Depending on the use of road capacity (workload), B. Beukers has determined three conditions: steady flow, unsteady flow, forced traffic (see Figure 1). The calculations have been performed for a road with the capacity of 2000 cars per hour in one lane [6].

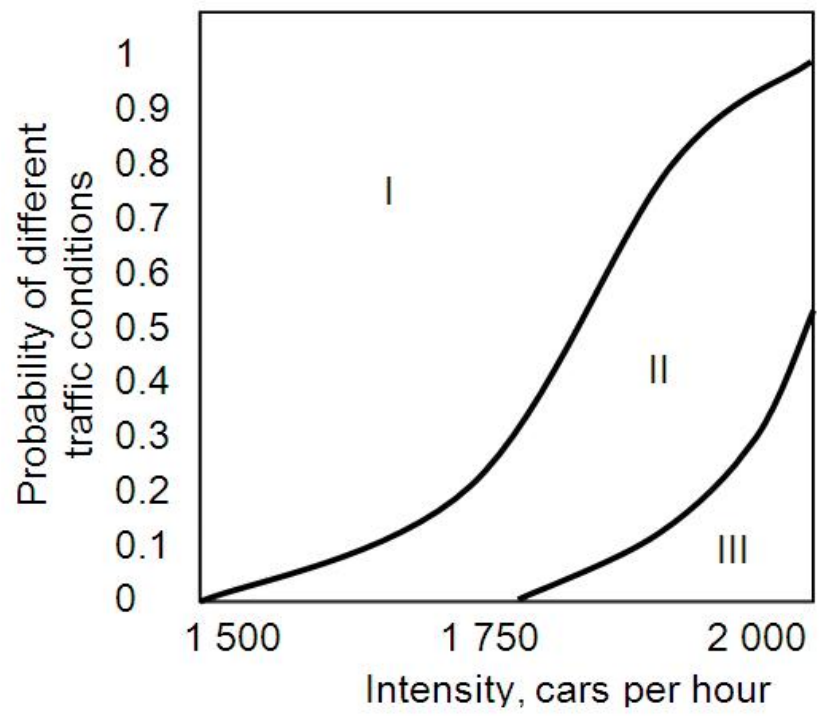

Figure 1. Relation between the intensity of flow intensity and traffic conditions:

$$
\begin{aligned}
& \text { I - steady flow; } \\
& \text { II - unsteady flow; } \\
& \text { III - forced traffic }
\end{aligned}
$$

Under the $75 \%$ load (with the intensity of 1500 cars per hour), the traffic flow starts to become unstable. As far as the intensity increases, the instability grows. The probability of an unsteady flow is $50 \%$ under $90 \%$ load. With 2000 light motor vehicles per lane, forced traffic emerges with a 50\% probability.

The most valuable part of this single-criterion model is the multidimensional representation of a traffic flow condition. In other words, in this case, the transition from one flow condition to another is defined not only by the change in traffic intensity, but also by the probability of every condition for each level of traffic flow intensity. For example, under high traffic intensity (2 000 cars per hour) any of the traffic flow condition (steady, unsteady, forced traffic) can occur with the following probabilities: 0.5 for forced traffic, 0.45 for unsteady traffic, 0.05 for steady traffic. Possible conditions form a set of exhaustive events.

The quality of a traffic flow is also characterized by six so called "service levels", depending on the type of the road, road capacity usage and actual speed [6].

For each service level, a traffic speed range for corresponding traffic intensity interval is defined. Within this problem statement, the model of service levels is a multi-criteria model. A service level is defined by the intensity and the average speed of traffic, which, in turn, is treated as a function of the flow intensity.

«Traffic convenience level» model, developed by V.V. Silyanov [3], also belongs to multi-criteria models. According to his data, every convenience level is characterized by certain drivers labor conditions, comfort and efficiency of transportation, as well as a certain accident rate.

Driving convenience levels are defined depending on the values of load, speed and traffic saturation level coefficients. On the whole, 4 traffic convenience levels are defined: «free», «partially bound», «bound» and «congested (saturated)». 
The class of multi-criteria concepts conditions of traffic flow comprise Kerner's theory three phases in which the flow condition is characterized by the intensity and speed. Besides phase free traffic flow Kerenr distinguish in phase congested traffic two phases: synchronized flow and wide moving jam, and explains the mechanisms of transition between them [12].

«Traffic convenience level» flow condition model provides a more comprehensive reflection the of qualitative changes in the flow that are caused by the mutual influence of basic flow parameters. Exemplifying classification of the aforementioned models is shown at Figure 2.

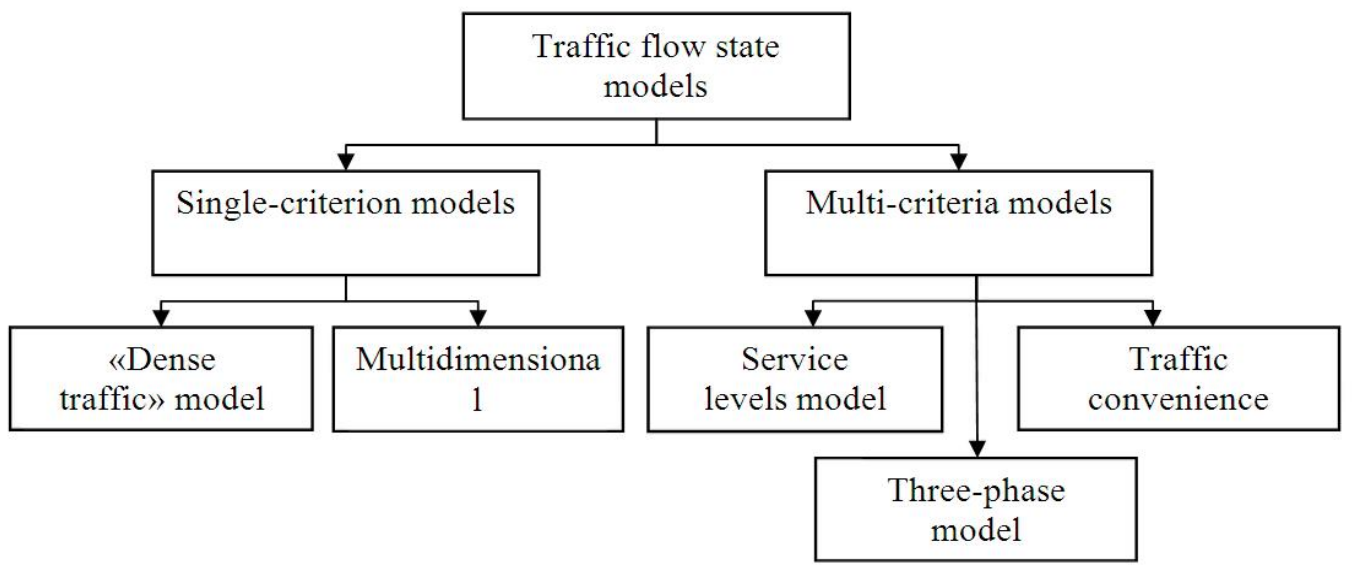

Figure 2. Classification of traffic flow condition estimation models

\section{Phase transitions for traffic flow conditions}

Formation of the flow qualities can be represented as phase transitions where continuous traffic flow condition changes being figuratively divided into several discrete (phase) conditions that form a phase space $[7,11]$.

During a phas e transition, a condition (phase) of a traffic flow changes due to the change in external conditions - that is, intensional parameters (i.e. density, distance, etc.) transmit the phase line. A step change in extensional parameters (i.e. speed, traffic intensity, etc.) occurs in the phase-transition point. As long as the phases are described by different equations of condition, it is always possible to find a value that changes stepwise during the phase transition.

Phase transitions of the first and the second kind can be defined. A phase transition of a first kind implies the change of the aggregate condition of the system. In this case, either the essential parameters of the system change stepwise, or the operation principles of the system change. The following two aggregate conditions of a traffic flow are defined: free and congested traffic. Each condition can be characterized through the possible choice of road speed. In free traffic, a driver can choose convenient speed, $v_{e}-$ «free» speed (so called «desired» speed). This speed approximates the maximum possible speed $\left(v_{e} \approx v_{\max }\right)$ under given traffic conditions: safe speed for a single car, speed limit defined by traffic rules, technically possible speed, etc. In heavy traffic, the speed $v_{e}$ is limited by the flow parameters (i.e. high flow density or heavy traffic, etc.), so the drivers choose the average speed of the flow $\left(v_{\mathrm{e}} \approx v_{\mathrm{e}}^{\prime}\right)$ that can be significantly lower than the maximum possible speed $\left(v_{\mathrm{e}}^{\prime}<v_{\max }\right)[8]$.

Phase transitions of the second kind are considered to be inappreciable to an unaided eye and to occur within the limits of the aggregate conditions. Stepwise changes can occur, for example, in first order derivatives of extensive characteristics, for example, first derivative of speed is acceleration or deceleration. Phase changes of the second kind can be found in the traffic flow conditions for free and congested traffic. For example, the phase conditions can be determined in free traffic depending on the braking of a car if a car ahead has used emergency braking.

The transition between the aggregate conditions is related to the limitation of speed by flow factors. Traffic speed is a determined (descending) function of a flow density: $v_{e}=v_{e}(\rho)$. With maximum flow density, $\rho_{\max }$, traffic is blocked, in other words, $v_{e}\left(\rho_{\max }\right) \approx 0$.

In accordance with the statements of follow-the-leader theory, all drivers adapt their speed, $v_{n}$, to the speed of the leader, $v_{n-1}$, while keeping the safe distance (depends on the speed) to the leader car $d_{s}\left(v_{e}\right)[9]$. 
The distance between the cars can be represented through the flow density:

$d_{\rho}=d_{e}+d_{\min }=1 / \rho-l_{a}$,

where $d_{e}$ is a dynamic distance; $d_{\min }$ is the minimal distance for maximum flow density; $l_{a}$ being the average car length in traffic.

For the maximum flow density, $\rho_{\max }$, the safe distance is $d_{s}=0$, because the speed $v_{e}=0$. In the first place, the increase of $\rho$ (flow density) leads to the decrease of maximum possible dynamic distance between cars $\left(d_{e}\right)$ to the value defined using (1) minus $d_{\min }$ :

$d_{e}=d_{\rho}-d_{\min }$,

and, consequently, to the possible decrease of safe distance $\left(d_{S}\right)$ and, as a consequence, to the possible limitation of car speed in traffic $\left(v_{e}\right)$ not by the maximum speed $\left(v_{\max }\right)$, but the safe speed $\left(v_{s}\right)$ with current safe distance: $v_{e} \leq v_{s}\left(d_{s}\right)$. By the data, $v_{s} \leq v_{\max }$.

This being said, the speed $\left(v_{e}\right)$ may not decrease, if the dynamic distance is not lower than safe distance for the speed, $v_{e}: d_{e} \geq d_{s}\left(v_{e}\right)$. Safe distance can be calculated according to the formula:

$d_{s} \geq v_{e} \tau+\Delta l_{m}+d_{\min }$.

where $\tau$ is the time of the reaction on the braking of a leader car; $\Delta l_{m}$ is the difference between the braking distance of the front and the rear car.

The study [2] considers some additional factors in the calculation of the distance, which are related to the perception of road events by the drivers, for example, the driver's sensitivity to the change of the leader's speed. These factors present some features of interest, as the congested flow traffic observations show that the majority of cars are moving with the lesser distance than is calculated according to (3). Moreover, the difference between the actual distance and the distance that was calculated using (3), increases as far as the traffic speed increases.

\section{Mechanism of phase transitions between traffic flow conditions}

Phase transitions between the flow conditions are caused by the establishment mechanism of car speed, $v_{e}$, as a function of the flow density. Dynamic distance between the cars cannot exceed the distance defined by the flow density conditions $\left(d_{e}<d_{\rho}\right)$, and the safe distance should not exceed the dynamic distance $\left(d_{s} \leq d_{e}\right)$ :

$d_{s} \leq d_{e}<d_{\rho}$.

For every $v_{\max }$ value, there is a safe distance $d_{s}\left(v_{\max }\right)$ calculated according to (3). The condition, $d_{s}\left(v_{\max }\right)>d_{e}$ means that the distance $d_{s}\left(v_{\max }\right)$, which is necessary for maximum speed, cannot be reached in accordance with the flow density conditions. For this reason, maximum safe distance, $d_{s}$, is defined not by the speed in accordance with (3), but by the flow density in accordance with (2). Subsequently, maximum possible speed, $v_{e}$ will be limited by $v_{\rho}$, which complies with the safety requirements in case of $d_{s}=d_{e}$ and is defined by the expression (3).

Limitation mechanism for the flow speed, $v_{e}$, is shown at the Figure 3, where the following graphics of monotonous functions are presented: $d_{s}$ is increasing function by speed, $d_{e}$ is decreasing by density. Maximum density, $\rho_{\max }$ can be defined using (1) if $\rho=\rho\left(d_{\rho}\right)=\rho(0)$.

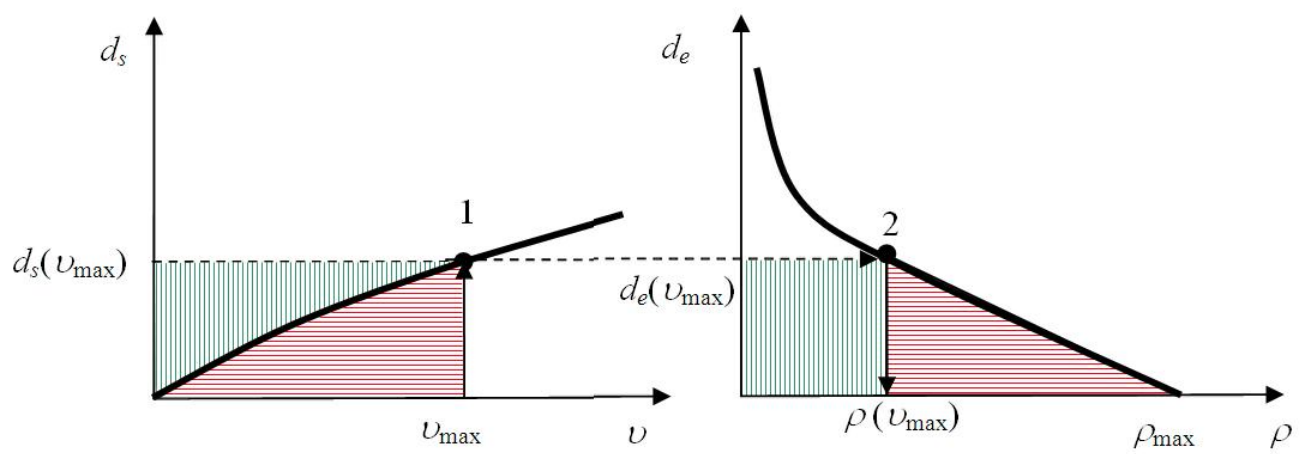

Figure 3. Establishment mechanism for traffic flow characteristics and conditions 
If road conditions allow the traffic speed to be equal $v_{\max }$, then, for safe driving at this speed, the distance $d_{s}\left(v_{\max }\right)$ should be kept - see point 1 (Figure 3 ). The condition (4) implies that this distance should not exceed the distance $d_{s}\left(v_{\max }\right) \leq d_{e}\left(\rho_{e}\right)$ that is defined by the flow density - see point 2 . Distance defined by density, $d_{e}\left(\rho_{e}\right)$ will be kept if the flow density does not exceed $\rho_{e}$. For this reason, the flow speed can reach the $v_{\max }$ value if the flow density at the section equals $\rho=\left(0, \rho_{e}\right)$. Hatched area shows the $\rho$ value that enables the driving with $v_{\max }$ speed. At the corresponding section, the flow condition is defined as free traffic.

The flow density increase at the section: $\rho=\left(\rho_{e}, \rho_{\max }\right)$ leads to the decrease of maximum possible speed, $v_{\max }$, which is set with regard to the limitation caused by the road factors. Flow condition for $\rho=$ $\left(\rho_{e}, \rho_{\max }\right)$ is defined as congested traffic.

Phase condition diagram of traffic flow conditions is shown at Figure 4. Driving distance $d_{\mathrm{e}}$ is considered to be the main intensive parameter. Traffic speed $v_{e}$ is considered to be the extensive parameter.

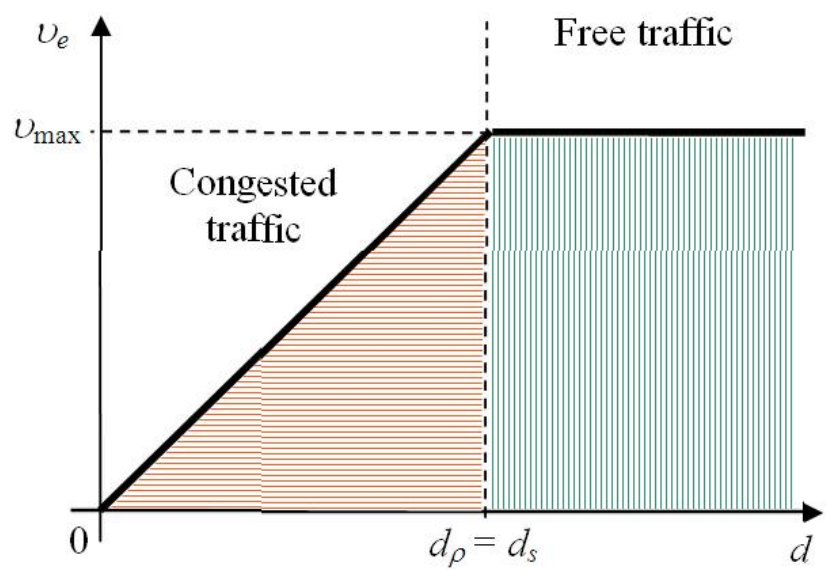

Figure 4. Phase condition diagram of traffic flow conditions

The diagram shows the aforementioned rules for phase transitions between traffic flow conditions. In the congested traffic area, the following condition is met: $d_{\rho}<d_{s}$. Consequently, the flow speed, $v_{e}$ is limited by the distance defined by density and is less than the maximum speed, $v_{\max }$, which is defined by the road conditions. When the distance defined by the flow density $d_{\rho}$ increases, the speed can also be increased if the road safety conditions are met (increasing function). In the free traffic area, the following condition is met: $d_{\rho}>d_{s}$. For this reason, the flow speed is limited by the possible road conditions, $v_{\max }=$ const (straight line).

It is worth mentioning that the phase diagram is similar to sectionally continuous Grinberg function, «speed - density», is shown at Figure 5 [10]. In accordance to this function, the flow moves «freely» with the average speed of $v_{c B}$ at a low density section $\left(0, \rho_{a}\right)$. When the density increases $\left(\rho_{0}>\rho_{a}\right)$, traffic speed starts to decrease, and for maximum density $\rho_{\max }$ the flow movement is impossible $(v=0)$.

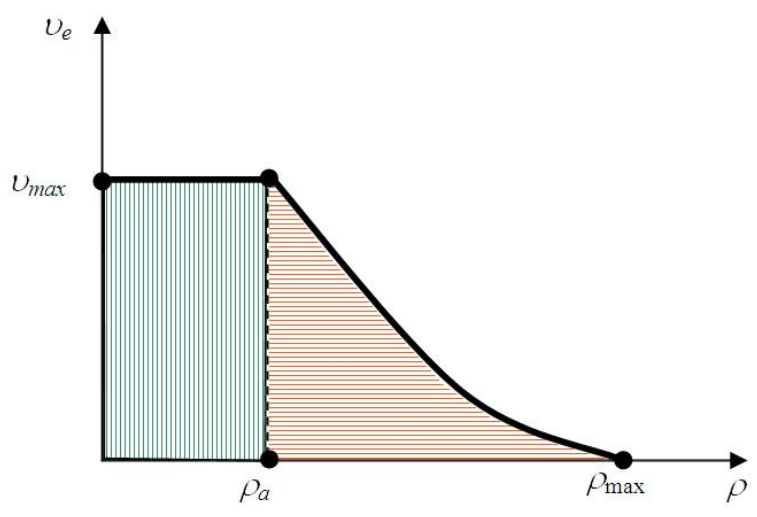

Figure 5. Greenberg's model 


\section{Model of the aggregate conditions of matter for a traffic flow}

The indicators of the flow conditions are: maximum possible speed under given road conditions, $v_{\max }$, (is the limitation of the flow speed, $v_{e}$ ); flow density, $\rho$, which is defined by experiment or by calculation; safe distance, $d_{s}$, which is calculated using (2); distance defined by the flow parameters, $d_{\rho}$, which is calculated using (1).

Aggregate condition of free traffic. It shall be assumed that the traffic flow is in the free movement condition if the cars can move with the maximum speed possible, $v_{\max }$. This condition is true for a particular flow density value, $\rho$, which makes the following inequality valid: $d_{e} \geq d_{s}\left(v_{\max }\right)$.

Aggregate condition of congested traffic. The increase of $\rho$ leads to the decrease of $d_{e}$. As soon as the relation between the distances change $\left(d_{e}<d_{s}\left(v_{\max }\right)\right)$, traffic speed, $v_{e}$, will be limited by the flow factors. The condition of the flow will change from free traffic to congested traffic.

In free traffic, the limit of the flow speed increase is defined by the $v_{\max }$ (limited by the road factors), in congested traffic the speed, $v_{e}$ is set at the level that guarantees safe traffic conditions for the distance that is limited by density, $d_{e}$ (limited by the flow factors):

$v_{e}=\left\{\begin{array}{ll}v_{\max }, & \text { if } d_{s} \leq d_{e} \\ v_{\rho}, & \text { if } d_{s}>d_{e}\end{array}\right.$.

This model generally characterizes the formation conditions for the conditions of homogeneous flow as a system in equilibrium. For this system, the identity of all its dynamic characteristics (density, speed, distance, etc.) holds true at a certain section of the road. It is usually considered that such properties are atypical for real conditions. In addition, the homogeneous properties of the flow are observed in congested traffic and heavy load, when all vehicles are moving with equally low speed, keeping small distance between the vehicles. The properties of a homogeneous flow are observed at the multi-lane roads, in particular, with two lanes for each direction, where the laminar effects are observed with the distribution of the cars along the lanes with regard to optimum speeds.

For this reason, phase transition model can be used with further modification in the light of specific features of traffic flow movement in particular transport networks.

\section{Conclusion}

Revealing the conditions of a traffic flow is highly important to estimate the measures taken to augment the transit potential of transport systems. To increase the speed of transit traffic in free traffic conditions, the roads can be broadened, the road surface can be repaired, etc. To increase the traffic speed in congested traffic, transport network reconfiguration should be considered.

\section{Acknowledgements}

The project is supported by the International Transport Academy (Russia), the parent organization of the Russian International Congress on Intelligent Transport Systems.

\section{References}

1. Larin, O.N. (2007). Methodology of organization and the functioning of regional transportation systems. Chelyabinsk: SUSU. - 205 p.

2. Daganzo, C.F. (1999). Possible explanations of phase transitions in highway traffic / C.F. Daganzo, M.J. Cassidy, R.L. Bertini, Transportation Research, Part A, V. 33, pp. 365-379.

3. Silyanov, V.V. (1977). Theory of traffic flows in road design and the organization of traffic. Moscow: Transport. 303 p.

4. Daganzo, C.F. (2002). A behavioral theory of multi-lane traffic flow. Part I: Long homogeneous freeway sections, Transportation Research, Part B, Vol. 36, pp. 131-158.

5. Daganzo, C.F. (2011). On the macroscopic stability of freeway traffic, Transportation Research, Part B, Vol. 45, pp. 782-788.

6. Stenbrink, P.A. (1974). Optimization of transport networks. London, New York, Sydney, Toronto: John Wiley\&Sons. 284 p. 
7. Gufan, J.M. (1982). Thermodynamic theory of phase transitions. Rostov na Donu: Rostov University Publishing House. 172 p.

8. Prigogine, I. A. and Andrews F.C. (1960). Boltzmann-like approach for traffic flow, Oper. Res., Vol. 8, pp. 798-797.

9. Shvetsov, V.I. and Aliyev, A.S. (2003). Mathematical modeling of the transport network boot. Moscow: Editorial URSS. 64 p.

10. Lukanin, V.N., Buslaev, A.P. and Yanshina, M.V. (2001). Transport flows and environment - 2. Moscow: INFRA-M. 646 p.

11. Kerner, B.S. and Rehborn, H. (1997). Experimental properties of phase transitions in traffic flow. Phys. Rev. Lett., Vol. 79, pp. 4030-4033.

12. Kerner, B. S., Klenov, S. L. and Hiller, A. (2006) Criterion for traffic phases in single vehicle data and empirical test of a microscopic three-phase traffic theory, J. Phys. A: Math. Gen., Vol. 39, pp. 2001-2020. 